\title{
A Low Overhead Checkpointing Protocol for Mobile Computing Systems
}

\author{
Chi-Yi Lin, Szu-Chi Wang, Sy-Yen Kuo \\ Department of Electrical Engineering \\ National Taiwan University \\ Taipei, Taiwan \\ sykuo@cc.ee.ntu.edu.tw
}

\author{
Ing-Yi Chen \\ Department of Electronic Engineering \\ Chung Yuan Christian University \\ Chung-Li, Taiwan \\ ichen@cycu.edu.tw
}

\begin{abstract}
Checkpointing protocols for distributed computing systems can also be applied to mobile computing systems, but the unique characteristics of the mobile environment need to be taken into account. In this paper, an improved time-based checkpointing protocol is proposed, which is suitable for mobile computing systems based on Mobile $I P$. The main improvement over a traditional time-based protocol is that our protocol reduces the number of checkpoints per checkpointing process to nearly minimum, so that fewer checkpoints need to be transmitted through the bandwidth-limited wireless links. The proposed protocol also performs very well in the aspects of minimizing the number and the size of messages transmitted in the wireless network. Therefore, the protocol brings very little overhead to a mobile host which has limited resource. Additionally, by integrating the improved timer synchronization technique, our protocol can also be applied to wide area networks.
\end{abstract}

\section{Introduction}

The infrastructure supporting mobile computation is growing mature rapidly. Users with mobile devices are able to access and exchange information on the move. As a result, collaborative works can be done effectively, no matter where the participating members/hosts are physically located. For example, in a sensor network which carries out real-time scientific computation, sensors with processing capability can be mobile and distributed.

To provide fault-tolerance capability for mobile computing systems, checkpointing and rollback-recovery techniques for traditional distributed computing systems such as [1, 2] can be used. Recently, checkpointing protocols specifically designed for mobile computing systems have also been proposed [3-11]. A common goal of these protocols is to avoid extra coordinating messages and unnecessary checkpoints. Prakash and Singhal [4] first proposed a checkpointing protocol that requires only a minimum number of processes to take checkpoints and does not block the underlying computation during checkpointing. However, Cao and Singhal [6] proved that such a min-process nonblocking checkpointing algorithm does not exist. They also introduced the concept of mutable checkpoints [10] in their nonblocking algorithm, which forces a minimum number of processes to take checkpoints on the stable storage.

Time-based protocols $[2,5,12]$ use synchronized clocks or timers to indirectly coordinate the creation of checkpoints so that coordinating messages are reduced. However, time-based protocols require every process to take a checkpoint during a checkpointing process. Moreover, since timers cannot be perfectly synchronized, the consistency between all the checkpoints can still be a problem. In [12], the problem is solved by disallowing message sending during a period after a timer expires, but doing this blocks the computation. In [5], however, processes are nonblocking because the inconsistency was resolved by the information piggybacked in each message. Timer synchronization can also be done using the piggybacked information. But when the transmission delay between two mobile hosts becomes relatively large, the synchronization result will be less accurate.

In this paper, we propose an improved time-based checkpointing protocol that tries to reduce the number of checkpoints. The basic idea is that if a checkpoint initiator does not transitively depend on a process, the process does not have to take a checkpoint associated with the initiator. The result is that the number of checkpoints transmitted over the air can be minimized. Also, and the number of coordinating messages is very small compared to other existing protocols. The protocol is also nonblocking because the inconsistency between processes is avoided by piggybacking necessary information in each message.

The rest of this paper is organized as follows. Section 2 describes the system model. In Section 3 we show the improved timer synchronization technique for time-based protocols. In Section 4 we present our checkpointing protocol and give a performance analysis. Section 5 concludes our work. 


\section{System Model and Background}

A mobile computing application is executed by a set of $N$ processes running on several mobile hosts (MHs). Processes communicate with each other by sending messages. These messages are received and then forwarded to the destination host by the mobile support stations (MSSs), which are interconnected by a fixed network. The mobility of $M H \mathrm{~s}$ is supported by Mobile IP, so that messages can be routed to the destination $M H$ which is moving around in the network. A $M H$ is associated with a Home Agent (HA)/Foreign Agent (FA) when it is in the home/foreign network.

To ensure ordered and reliable message deliveries, each message is assigned an increasing sequence number. In the system every process takes a checkpoint periodically. Each checkpoint is associated with a monotonically increasing checkpoint number. The time interval after taking the $k^{\text {th }}$ checkpoint and before taking the $k+1^{\text {th }}$ checkpoint is called the $k^{\text {th }}$ checkpoint interval (represented as $I_{k}$ in the following text).

In the system every node (MH or MSS) contains a system clock, with typical clock drift rate $\rho$ in the order of $10^{-5}$ or $10^{-6}$. The system clocks of MSSs can be synchronized using Internet time synchronization services such as Network Time Protocol, which makes the maximum deviation $\sigma$ of all the clocks within tens of milliseconds. However, in wide area networks, MSSs may belong to different organizations. So, we use the clock synchronization protocol to sync the logical clocks instead of the physical system clocks of MSSs. The clocks of $M H \mathrm{~s}$ can be synchronized likewise, but explicit synchronization messages bring overhead to $M H \mathrm{~s}$ because of the limited wireless bandwidth. In addition, the system clocks of $\mathrm{MHs}$ may not be controlled by a user-level application. Therefore, to coordinate with each other, processes use synchronized timers instead of synchronized clocks. The advantages of using timers to coordinate the creation of checkpoints are that the checkpointing protocol does not have to rely on synchronized system clocks, and no explicit synchronization is needed.

Before a mobile computing application starts, a predefined checkpoint period $T$ is set on the timers. When the local timer expires, the process saves its system state as a checkpoint. If all the timers expire at exactly the same time, the set of $N$ checkpoints taken at the same instant forms a globally consistent checkpoint. Since timers are not perfectly synchronized, the checkpoints may not be consistent because of orphan messages. An orphan message $m$ represents an inconsistent system state with the event receive $(m)$ included in the state while the event $\operatorname{send}(m)$ not in the state. Orphan messages may lead to domino effect, which causes unbounded, cascading rollback propagation. So, by definition, a globally consistent checkpoint is free from the domino effect.

\section{Improved Timer Synchronization}

In this section we introduce the mechanism of improved timer synchronization. The mechanism then serves as a basis in our checkpointing algorithm, as described in the next section.

The mechanism of timer synchronization in [5] uses piggybacked timer information from the sender to adjust the timer at the receiver. When the sender sends a message, it piggybacks its "time to next checkpoint" (represented as timeToCkp) in the message. The receiver then uses the information to adjust its own timeToCkp. The checkpoint number of the sender is also piggybacked in the message, so that the receiver can act accordingly to avoid an orphan message. However, if the timer of the sender is faulty, the erroneous timer information will be spread to the receiver. Besides, since the transmission delay between the sender and the receiver is variable, the timer information from the sender may not reflect the correct situation when the message finally arrives at the receiver.

To achieve more accurate timer synchronization, we utilize the timers in MSSs as an absolute reference because timers in the fixed hosts are more reliable than those in $M H$ s. We also assume that the timers of the MSSs are synchronized every checkpoint period. In our design, the local MSS of the receiver is responsible for piggybacking its own timeToCkp in every message destined to the receiver, because the MSS is the closest fixed host to the receiver.

In the system every $M H / M S S$ maintains a checkpoint number. In the following we use $c n_{S}, c n_{D}$, and $c n_{M S S}$ to represent the checkpoint number of the sender, the receiver, and the local MSS of the receiver, respectively. Like [5], the sender piggybacks its own checkpoint number $\mathrm{cn}_{S}$ in each message. When the local MSS of the receiver receives the message, apart from timeToCkp, it also piggybacks $c n_{M S S}$ in the message, and then it forwards the message to the receiver. So, when receiving the message, the receiver has the following information: $c n_{S}, c n_{M S S}$, and timeToCkp of the local MSS (represented as m.timeToCkp). Note that in practice messages take a minimum time $t d_{\min }$ to be delivered from a $M S S$ to a $M H$ in its cell. So, whenever the local timer of a $M H$ is adjusted by m.timeToCkp, substracting $t d_{\min }$ from m.timeToCkp makes the adjustment more accurate. In the following description we use the symbol $\Delta$ to represent minus $t d_{\min }$. The relationship between $c n_{D}, c n_{M S S}$, and $c n_{S}$ determines how the timer is adjusted, as described in the following cases.

I. $c n_{S}=c n_{D}$

(1) $c n_{M S S}=c n_{S}=c n_{D}:$ The receiver resets its timeToCkp to "m.timeToCkp $+\Delta$ ".

(2) $c n_{M S S}>c n_{S}=c n_{D}$ : The timer of $M H_{D}$ is late compared to that of $M S S_{2}$. So as soon as message $m$ is processed, $M H_{D}$ takes a checkpoint with ckpt number $c n_{M S S}$, and then resets its timeToCkp to "m.timeToCkp $+\Delta$ ". 
(3) $c n_{M S S}<c n_{S}=c n_{D}$ : The timers of $M H_{S}$ and $M H_{D}$ are both early compared to that of $M S S_{2}$. $M H_{D}$ resets its timeToCkp to " $T+$ m.timeToCkp $+\Delta$ ".

II. $c n_{S}<c n_{D}$

(1) $c n_{S}<c n_{M S S}=c n_{D}$ : Since $M H_{D}$ and its local MSS are within the same ckpt period, $M H_{D}$ just resets its timeToCkp to "m.timeToCkp $+\Delta$ ".

(2) $c n_{S}=c n_{M S S}<c n_{D}: c n_{M S S}<c n_{D}$ means that the timer of $M H_{D}$ expires too early, so $M H_{D}$ resets its timeToCkp to " $T+$ m.timeToCkp $+\Delta$ ".

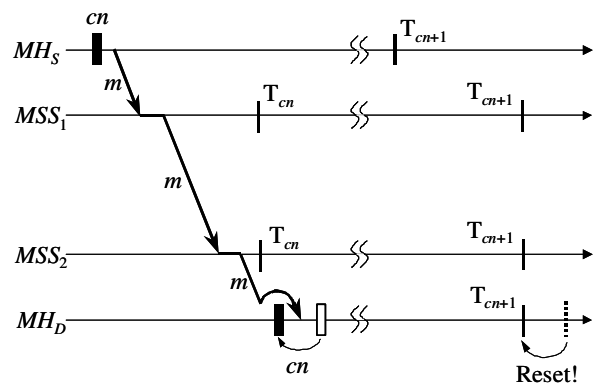

(a)

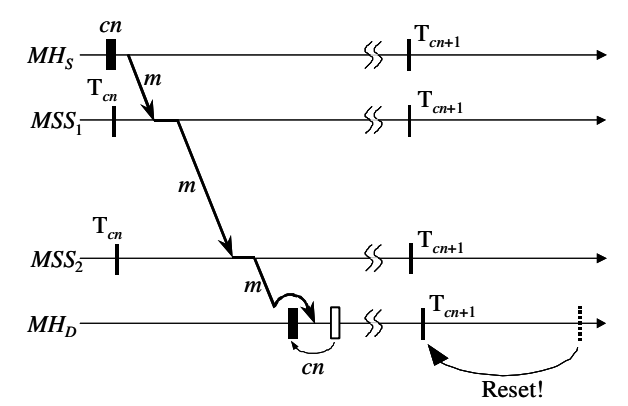

(b)

Figure 1. Timer synchronization (a) $c n_{S}>c n_{M S S}=c n_{D}$ (b) $c n_{S}=c n_{M S S}>c n_{D}$.

III. $c n_{S}>c n_{D}$

(1) $c n_{S}>c n_{M S S}=c n_{D}$ (Fig. 1(a)): Before $M H_{D}$ can process $m$, it has to take a ckpt with ckpt number $\mathrm{Cn}_{S}$; otherwise $m$ is an orphan message. Then $M H_{D}$ resets its timeToCkp to " $T+$ m.timeToCkp $+\Delta$ ".

(2) $c n_{S}=c n_{M S S}>c n_{D}$ (Fig. 1(b)): $M H_{D}$ has to take a ckpt before processing $m$ in order not to make $m$ an orphan message. Since the timer of $M H_{D}$ is late compared to that of $M S S_{2}\left(c n_{M S S}>c n_{D}\right), M H_{D}$ then resets its timeToCkp to "m.timeToCkp $+\Delta$ ".

From the above discussion, we can find that the receiver's timer can be synchronized whenever a message is received. Since the synchronization information is piggybacked in every message, the sender's timer can also be synchronized with its local MSS as soon as the sender receives the acknowledgement.

In the next section, our checkpointing protocol requires that at the end of a checkpoint interval, none of the $M H$ 's timers expires earlier than those of MSSs. To fulfill the requirement, we need to take the clock drifts of $M H$ s and MSSs into account. The clock drift rates of the timers in $M H \mathrm{~s}$ and $M S S \mathrm{~s}$ are represented as $\rho_{M H}$ and $\rho_{M S S}$ respectively. In the system model we also mentioned that after the clock synchronization, there exists a maximum deviation $\sigma$ between two MSSs. In the following lemma, we show how the requirement is achieved.

Lemma 1: By setting $\Delta=\sigma+2 \rho_{M S S} \times T+\rho_{M H} \times 2 T-t d_{\text {min }}$ in the algorithm, $\forall$ process that has received a message in $I_{c n-1}$, its $I_{c n+1}$ begins no earlier than that of a MSS.

Proof: Assume a process is in $I_{c n-1}$ and it receives a message. It is straightforward that the maximum time deviation between any two MSSs after a time period $T$, is $\sigma+2 \rho_{M S S} \times T$. If receiving the message triggers a new ckpt to be taken immediately, the maximum time to the $c n+1^{\text {th }}$ ckpt is $2 T$. As a result, the maximum time deviation between the process and its $M S S$ is $\rho_{M H} \times 2 T-t d_{\min }$ from receiving the message to taking the $\mathrm{cn}+1^{\text {th }} \mathrm{ckpt}$. By setting $\Delta=\sigma+2 \rho_{M S S} \times T+\rho_{M H} \times 2 T-t d_{\min }$, the adjustment of timeToCkp makes the local timer expire no earlier than that of a MSS for $I_{c n}$. On the other hand, if receiving the message does not trigger a new ckpt immediately, the maximum time to the $c n+1^{\text {th }} \mathrm{ckpt}$ is $T$. But multiplying $2 T$ with $\rho_{M H}$ in $\Delta$ ensures that even if the process does not receive any message during $I_{c n}$, the process's $I_{c n+1}$ will not begin earlier than that of a MSS.

\section{Time-based Checkpointing Protocol}

In this section, we present our time-based checkpointing protocol, which is applicable for mobile computing systems over Mobile IP.

\subsection{Notations and Data Structures}

- SoftCkpt ${ }^{c n}$ : The $c n^{\text {th }}$ soft checkpoint of a process, saved in the main memory of a $M H$.

- Perm $\mathrm{Ckpt}^{c n}$ : The $\mathrm{cn}^{\text {th }}$ permanent checkpoint of a process, saved in the stable storage of the process' $H A$ or FA. The system recovery line consists of $N$ consistent permanent checkpoints, one from each process.

- Cell $l_{k}$ : The wireless cell served by $M S S_{k}$.

- Recvi: An array of $N$ bits of process $P_{i}$ maintained by $P_{i}$ 's local MSS. In the beginning of every checkpoint interval, $\operatorname{Recv}_{i}[j]$ is initialized to 0 for $j=1$ to $N$, except that $\operatorname{Recv}_{i}[i]=1$. When $P_{i}$ receives a message $m$ from $P_{j}$, and the receipt of $m$ is confirmed by $P_{i}{ }^{\prime}$ s $M S S, \operatorname{Recv}_{i}[j]$ is set to 1 .

- LastRecvi: The $\operatorname{Recv}_{i}$ of the preceding checkpoint interval of process $P_{i}$, maintained by $P_{i}$ 's local MSS. 
- $C k p t N_{i}$ : The current checkpoint number of $P_{i}$ in the local MSS's knowledge.

- RejectCP $P_{i}$ A variable that saves a checkpoint number of process $P_{i}$, maintained by $P_{i}$ 's local MSS. When $P_{i}$ is trying to transmit its soft checkpoint to the $H A / F A$, the local MSS rejects the transmission if the checkpoint number of the soft checkpoint equals $\operatorname{Reject} P_{i}$.

\subsection{Checkpointing Protocol}

4.2.1. Checkpoint initiation. When the local timer expires, a process takes a soft checkpoint. More precisely, a soft checkpoint SoftCkpt ${ }^{c n}$ is taken at the beginning of $I_{c n}$. After a soft checkpoint has been taken, the process resumes its computation.

For simplicity, here we assume that only one of the $N$ processes will play the role of the checkpoint initiator of a checkpoint interval. Let's say process $P_{i}$ decides to act as the initiator of the next checkpoint interval. In the algorithm, $P_{i}$ has to send a checkpoint request to its local $M S S$ during the current checkpoint interval. On receiving the checkpoint request, the MSS becomes the initiator MSS (denoted by $M S S_{\text {init }}$ ), which is responsible for collecting and calculating the dependency relationship between the initiator and all other processes in the next checkpoint interval.

4.2.2. Maintaining dependency variables in MSSs. Since an MSS is responsible for forwarding messages for the processes in its cell, it is reasonable to use the MSS to maintain the dependency variables (Recv, LastRecv) for those processes as well. For example, process $P_{i}$ in $\mathrm{Cell}_{k}$ receives a message from $P_{j}$ and then sends an $A C K$ back to $P_{j}$ via $M S S_{k}$. By inspecting the $A C K, M S S_{k}$ knows that the message from $P_{j}$ has been delivered, so $M S S_{k}$ sets $\operatorname{Recv}_{i}[j]$ to 1 . Note that the $A C K$ is piggybacked with the checkpoint number of $P_{i}$ as described in Section 3, which can be used by $M S S_{k}$ to tell whether $P_{i}$ has entered the next checkpoint interval or not. As soon as $M S S_{k}$ finds that $P_{i}$ has entered a new checkpoint interval, $M S S_{k}$ saves the current $\operatorname{Recv}_{i}$ as LastRecv $v_{i}$, resets Recv $v_{i}$, and then modifies $R e c v_{i}$ accordingly. At the same time, $M S S_{k}$ also updates $\mathrm{CkptNum}_{i}$ for $P_{i}$. Note that the variable RejectCP is also maintained in the MSS, but the explanation is left to Section 4.3.2.

4.2.3. Determining the dependency relationship. As soon as the timer of $M S S_{\text {init }}$ expires, $M S S_{\text {init }}$ broadcasts a Recv_Request message to all MSSs. At $T_{\text {defer }}$ after receiving Recv_Request, each MSS sends to $M S S_{\text {init }}$ the dependency vector (Recv or LastRecv) of every process in its cell. Here $T_{\text {defer }}$ is a tunable parameter that the last message sent by a process before the process's timer expires is expected to arrive at the local MSS no later than $T_{\text {defer }}$ after the $M S S$ 's timer expires. We can choose a proper $T_{\text {defer }}$ according to the $Q o S$ requirements of the wireless network: the better the $Q o S$, the smaller the $T_{\text {defer }}$. A reasonable upper bound of $T_{\text {defer }}$ can be one half of a checkpoint period $(T / 2)$, which is normally in the order of several minutes or more.

After receiving all the dependency vectors, $M S S_{\text {init }}$ constructs an $N \times N$ dependency matrix $D$ with one row per process. We adopt the algorithm in [6] that by matrix multiplications, all the processes on which the initiator transitively depends can be calculated. In the following we call such processes initiator-depended processes. After finishing the calculation, the final dependency vector $D_{\text {init }}$ can be obtained, in which $D_{\text {init }}[i]=1$ represents that the initiator transitively depends on $P_{i}$ in the preceding checkpoint interval.

4.2.4. Discarding unnecessary soft checkpoints. A process can discard the newly taken soft checkpoint if the initiator does not transitively depend on the process in the preceding checkpoint interval. To do that, $M S S_{\text {init }}$ obtains a set $S \_$Discard ${ }^{\text {cn }}$ from $D_{\text {init }}$, which consists of any process $P_{i}$ such that $D_{\text {init }}[i]=0$, and then $M S S_{\text {init }}$ sends a notification DISCARD ${ }^{c n}$ to the processes in S_Discard ${ }^{c n}$. If process $P_{j}$ receives DISCARD ${ }^{c n}$, it deletes SoftCkpt ${ }^{c n}$ from its main memory, and the local MSS of $P_{j}$ sets Recv $=\left(\right.$ LastRecv $\left._{j} \vee \operatorname{Recv}_{j}\right)$.

On the other hand, if a process does not receive DISCARD ${ }^{c n}$ until $T_{\text {decide }}$ after taking SoftCkpt ${ }^{c n}$, it will send SoftCkpt ${ }^{c n}$ to a fixed host to make the checkpoint a permanent one. Here $T_{\text {decide }}$ is also a tunable parameter, which represents a reasonably long period of time from entering the current checkpoint interval to DISCARD ${ }^{c n}$ should have been delivered to all the processes in S_Discard ${ }^{c n}$.

4.2.5. Maintaining permanent checkpoints. In order to ensure the robustness of the recovery line, the soft checkpoints in a $M H$ 's memory should be transmitted to the stable storage of a fixed host periodically. In a mobile computing system based on Mobile IP, the stable storage of the home agent $(H A)$ or foreign agent $(F A)$ is an ideal place to store the permanent checkpoints for the processes. When an $H A / F A$ receives a soft checkpoint SoftCkpt ${ }^{c n}$ from process $P_{i}$, it saves SoftCkpt in its stable storage as a permanent checkpoint Perm Ckpt $t^{c n}$ of $P_{i}$. If SoftCkpt ${ }^{c n}$ of a process is discarded, the process's local MSS will inform the process's HA/FA to renumber PermCkpt ${ }^{c n-1}$ as PermCkpt ${ }^{c n}$ for the process. After the $H A / F A$ has collected all the checkpoints it should have received, it then proposes to advance the recovery line to checkpoint number $c n$. By adopting any feasible total agreement protocol for distributed systems, the recovery line will be committed to be advanced. 
4.2.6. Handling disconnections and handoffs. When an $M H$ within its $\mathrm{cn}^{\text {th }}$ checkpoint interval is about to disconnect with its local $M S S$ (say $M S S_{p}$ ), the processes on the $M H$ are required to take a soft checkpoint with checkpoint $c n+1$, and then send these checkpoints to $M S S_{p}$. Assume process $P_{i}$ takes a soft checkpoint SoftCkpt ${ }^{c n+1}$ and sends it to $M S S_{p}$. On receiving Soft $C k p t^{c n+1}, M S S_{p}$ saves (i, Soft $C k p t^{c n+1}$ ) in the stable storage, but $M S S_{p}$ does not forward SoftCkpt ${ }^{c n+1}$ to $P_{i}$ 's $H A / F A$ at the moment. The reason is that SoftCkpt ${ }^{c n+1}$ may possibly be discarded later if $P_{i}$ is in $S_{-}$Discard $^{c n+1}$. If $M S S_{p}$ finds that $P_{i}$ is not in $S_{-}$Discard $^{\text {cn+1 }}$, it sends SoftCkpt ${ }^{c n+1}$ to $P_{i}$ 's $H A / F A$ on behalf of $P_{i}$. Note that if the $M H$ is about to disconnect before $T_{\text {decide }}$ after entering the $c n^{\text {th }}$ checkpoint interval, $P_{i}$ has to send Soft $C k p t^{c n}$ along with SoftCkpt ${ }^{c n+1}$ to $M S S_{p}$. In this case, $M S S_{p}$ keeps SoftCkpt ${ }^{c n}$ for $P_{i}$ until $T_{\text {decide }}$ after entering the $c n^{\text {th }}$ checkpoint interval: $M S S_{p}$ may either discard it or send it to $P_{i}$ 's $H A / F A$, depending on $P_{i}$ is in $S \_$Discard ${ }^{c n}$ or not.

For a disconnected process, its dependency information (Recv, LastRecv, RejectCP, CkptNum) is still kept in the $M S S$. If the process reconnects with another MSS at a later time, the old MSS then sends the dependency information of the process to the new MSS. For the handoff of a $M H$, the old $M S S$ also forwards the dependency information of all the processes in the $M H$ to the new MSS. If the handoff involves a change of agents, the old agent forwards the permanent checkpoints of the processes in the $M H$ to the new agent.

In the following we present a formal description of our checkpointing algorithm:

\section{Action at the initiator $\boldsymbol{P}_{j}$ :}

01 send Checkpoint Request to the local MSS,
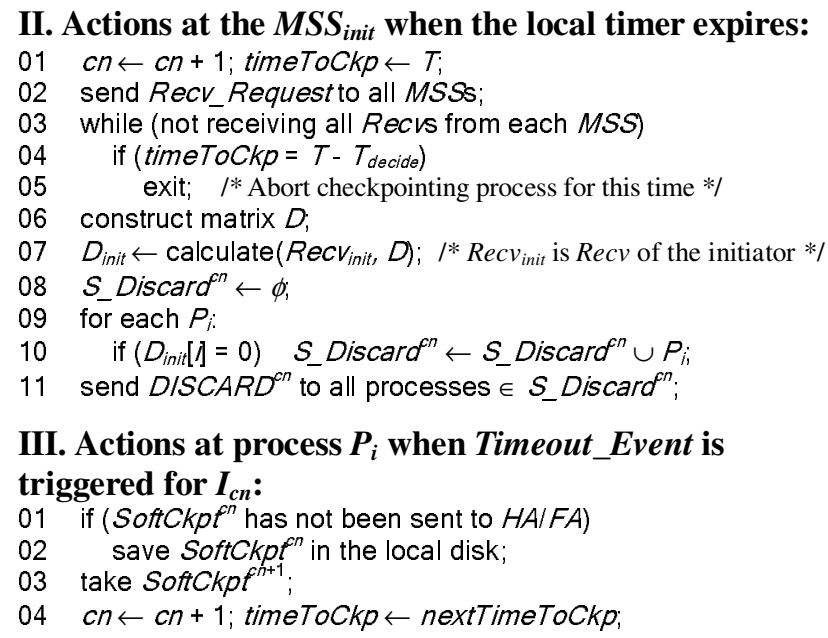

IV. Actions executed at an $M S S$, say $M S S_{k}$, in $I_{c n}$ : 01 upon relaying message $m$ from $P_{i} \in$ Cell $l_{k}$ to $P_{i}$ 02 if $\left(m . c n_{i}>C k p t N u m_{i}\right)$ \{

$03 \quad$ CkptNum $\leftarrow$ m.cni, LastRecvi $\leftarrow$ Recvi, reset Recvi, 04 05

V. modify $R e c v_{i}$ if necessary, then send $m$ to $P_{j}$ else if $\left(m . c n_{i}=C k p t N u m_{i}\right)$ modify $R e c v_{i}$ if necessary, then send $m$ to $P_{j}$;

else $\quad / * m . \mathrm{cn}_{i}<\mathrm{CkptNum}_{i}, m$ is an out-of-sequence message */ send $m$ to $P_{i}$;

upon receiving Recv_Request from $M_{S} S_{\text {init }}$

wait $\left(T_{\text {defer }}\right)$;

for each $i$ that $P_{i} \in$ Cellk:

if $\left(C K p t N u m_{i}=\mathrm{cn}\right)$ send LastRecvi to MSS $_{\text {init, }}$

else /*CkptNum ${ }_{i}<\mathrm{cn}$, and $\mathrm{CkptNum}_{i}$ cannot be larger than $\mathrm{cn} * /\{$ for any $j$ that a message from $P_{j}$ is unacknowledged: $\operatorname{Recv}[] \leftarrow 1$;

send Recvi to MSS $_{\text {init }}$ LastRecv $_{i} \leftarrow$ Recvi $_{i}$

\}

reset $\operatorname{Recv}_{i}$ CKptNum $_{i} \leftarrow c n_{i}$

upon receiving DISCARD ${ }^{c n}$ for $P_{i}$ in Cell $l_{k}$ from $M S S_{\text {init }}$

if ( $P_{i}$ is disconnected) discard SoftCkpf $f^{n}$ of $P_{i}$

else forward DISCARD ${ }^{\text {cn }}$ to $P_{i}$

$R_{e c v_{i} \leftarrow \text { LastRecv }_{i} \vee R e c v_{i}}$

upon receiving Disconnect_Request from $\mathrm{MH}_{q}$ in Cell

for each $P_{i}$ in $M H_{a}:$ I* Soft $C k p t^{c+1}$ is included in the request */ save SoftCkpf $f^{n+1}$ of $P_{i}$ in the local disk;

upon receiving Handoff Request from $\mathrm{MH}_{q}$ in Cell

for each $P_{i}$ in $M H_{q}$ : send $\left(\right.$ Recv $_{i,}$ LastRecv $_{i}$ CKptNum $_{i}$, RejectCP $\left._{i}\right)$ to the new MSS of $P_{;}$

upon $T_{\text {decide }}$ after entering the $c n^{\text {th }}$ checkpoint interval:

for any isuch that $D I S C A R D^{c n}$ for $P_{i} \in C e l_{k}$ is undelivered: RejectCP $\leftarrow$ icn;

upon receiving ForwardCP_Request( $(\mathrm{C})$ from $P_{i} \in$ Cell: if $($ RejectCP $\neq \mathrm{cm})$ receive and then forward the ckpt to the $H A / F A$ of $P_{i}$ else reject the transmission

upon expiration of the local timer:

$c n \leftarrow c n+1 ;$ timeTockp $\leftarrow T$

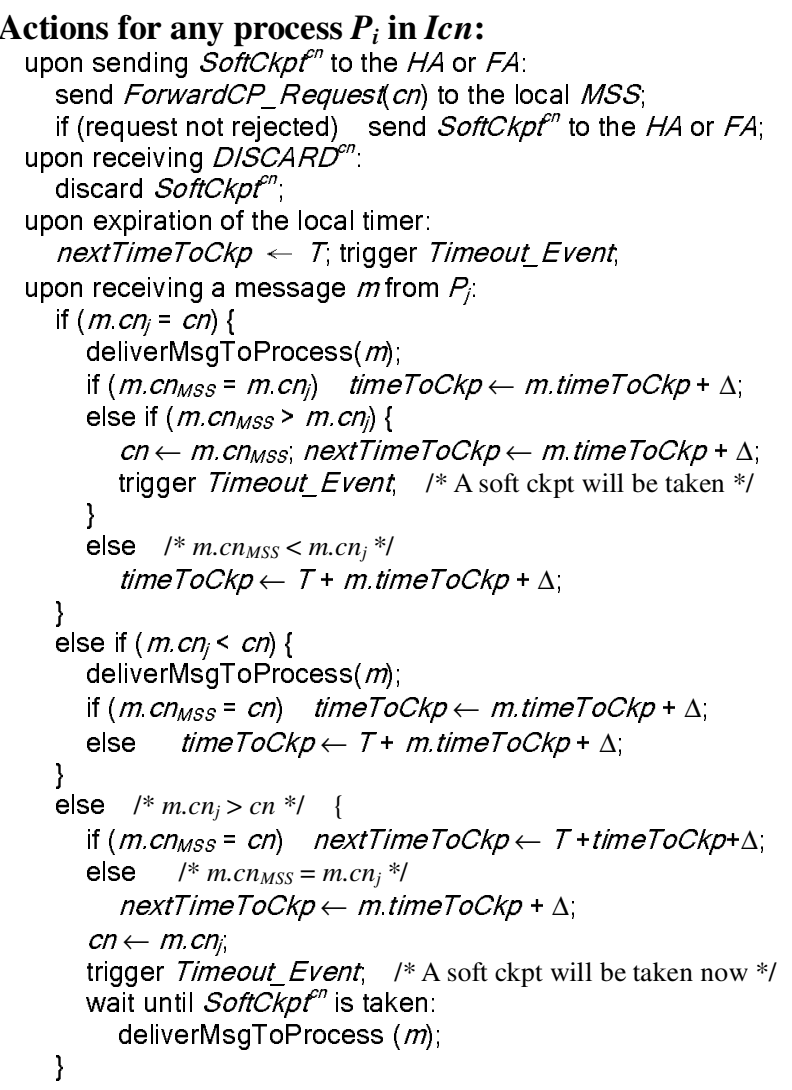




\subsection{Handling Untimely Delayed Messages}

In this section we discuss the problem of untimely delayed messages in the network. Since there exists inherent uncertainty of message delivery time in the wired and wireless network, we have to deal with untimely delayed messages in the checkpointing algorithm carefully.

4.3.1. Untimely delayed Recv vectors. When $M S S_{\text {init }}$ is collecting the Recv vectors, it is possible that because of network congestions or link failures in the wired network, some of the Recv vectors have not been received until $T_{\text {decide }}$ after entering the current checkpoint interval. In this case, the checkpointing process for this time has to be aborted (see code II of the checkpointing algorithm, lines 03-05). In effect, aborting the checkpointing process does not stop the progression of the recovery line since every process has taken a soft checkpoint, and these soft checkpoints will become permanent when they are sent to the $H A$ s or $F A$ s.

4.3.2. Untimely delayed DISCARD ${ }^{c n}$ notifications. An inconsistency situation may occur due to untimely delayed DISCARD ${ }^{c n}$ notifications. Although we can choose a proper $T_{\text {decide }}$ value such that the untimely delayed notifications are very rare, our algorithm has to cope with the problem in order to ensure the consistency of the global checkpoints. Let's demonstrate the problem as illustrated in Figure 2.

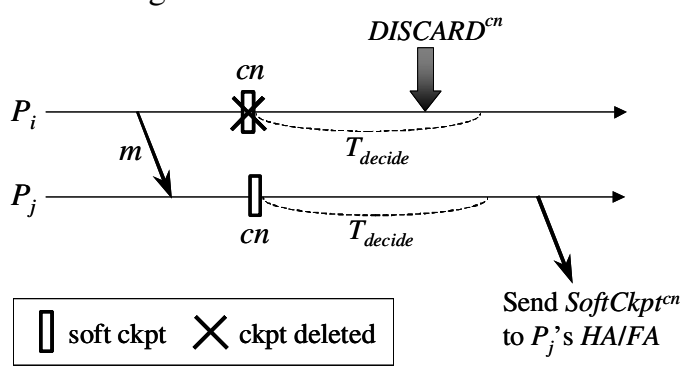

Figure 2. A possible scenario that the delivery of DISCARD ${ }^{c n}$ for $P_{j}$ is delayed.

Assume $P_{i}$ and $P_{j}$ are both in $S \_$Discard ${ }^{c n}$, but $P_{j}$ does not receive DISCARD ${ }^{c n}$ until $T_{\text {decide }}$ after entering $I_{c n}$. For $P_{i}$, since its $\operatorname{SoftCkpt}{ }^{c n}$ is discarded, its $H A / F A$ will renumber $P_{i}$ 's PermCkpt $t^{c n-1}$ as PermCkpt ${ }^{c n}$. For $P_{j}$, it will send its SoftCkpt ${ }^{c n}$ to its $H A / F A$ in order to make the checkpoint permanent. However, if there exists a message $m$ between $P_{i}$ and $P_{j}, m$ will be an orphan message with respect to $P_{i}$ 's PermCkpt ${ }^{c n}$ and $P_{j}$ 's Perm $C k p t^{c n}$. To cope with the problem, we introduce the variable Reject $C P$ of a process, which is also maintained by the local MSS of the process. In the above example, the local MSS of $P_{j}$ is aware that DISCARD ${ }^{c n}$ for $P_{j}$ has not been delivered until $T_{\text {decide }}$ after entering $I_{c n}$, so it sets $\operatorname{Reject} C P_{j}$ to $c n$. Afterwards when $P_{j}$ tries to send its SoftCkpt ${ }^{c n}$ to $H A / F A$, the $M S S$ rejects the transmission because $\operatorname{RejectCP}_{j}$ equals $c n$ (see code IV, lines 30-36). Therefore, $P_{j}^{\text {'s }}$ Perm $C k p t^{c n-1}$ will be renumbered as Perm $\mathrm{Ckpt}^{\mathrm{cn}}$ so that the inconsistency no longer exists. On $P_{j}$ 's part, if the transmission of its SoftCkpt ${ }^{c n}$ is rejected by the local MSS, $P_{j}$ deletes SoftCkpt $t^{c n}$.

4.3.3. Untimely delayed acknowledgements. In our algorithm, the MSS maintains the dependency vectors Recv and LastRecv for a process by inspecting the piggybacked information in an $A C K$ sent by the process, but an untimely delayed $A C K$ could be a problem during the checkpointing process. Take Figure 3 as an example, when $M S S_{k}$ is about to send $\operatorname{Recv}_{i}$ to $M S S_{\text {init }}$, ACK.m has not arrived so that $M S S_{k}$ cannot tell whether or not to include the receipt of $m$ in $\operatorname{Recv}_{i}$ at the instant. In our algorithm we take the following policy (refer to code IV, lines 14-19): when $M S S_{k}$ is about to send $\operatorname{Recv}_{i}$ to $M S S_{\text {init }}$ and it finds that such an unacknowledged message exists, $\operatorname{Recv}_{i}[j]$ is set to 1 . That is, $M S S_{k}$ presumes the case in Figure 3(a) always occurs. But if ACK.m finally arrives and shows that Figure 3(b) is true instead, the receipt of $m$ is then included in $\operatorname{Recv}_{i}$ of $I_{c n}$.

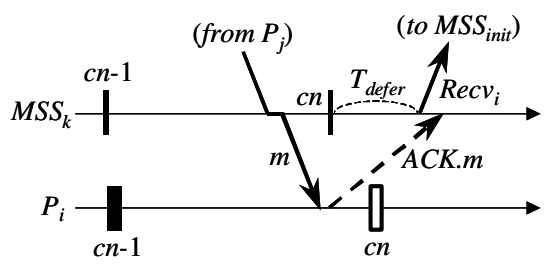

(a)

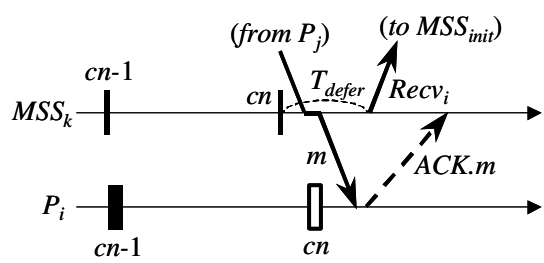

(b)

Figure 3. The $A C K$ of $m$ arrives later than $M S S_{k}$ has sent $R e c v_{i}$ to $M S S_{\text {init }}$ (a) Receipt of $m$ is in $I_{c n-1}$ of $P_{i}$ (b) Receipt of $m$ is in $I_{c n}$ of $P_{i}$.

\subsection{Rollback Recovery}

When a failure occurs, all the processes roll back to the latest recovery line. Assume the latest recovery line is numbered as $c n$. For a non-faulty process, if its SoftCkpt ${ }^{c n}$ is still in the main memory and its RejectCP is not $c n$, it can roll back to the state of SoftCkpt ${ }^{c n}$ because the content of SoftCkpt in identical to PermCkpt ${ }^{c n}$. Otherwise, the process requests its PermCkpt $t^{\text {cn }}$ from the $H A$ or $F A$.

From the above description, we can see that with the help of local soft checkpoints, some of the processes can be recovered locally so that the recovery can be done efficiently. 


\subsection{Proofs of Correctness}

Lemma 2. If a process $P_{i}$ receives a message from another process $P_{j}$ during $I_{c n-1}$ and $P_{j} \in S_{-}$Discard $^{c n}$, then $P_{i} \in S_{-}$Discard $^{\text {cn }}$.

Proof: If $P_{i} \notin S \_$Discard ${ }^{c n}$, from the proposed algorithm, the initiator transitively depends on $P_{i}$ during $I_{c n-1}$. Since $P_{i}$ depends on $P_{j}$, the initiator also transitively depends on $P_{j}$ during $I_{c n-1}$. From the proposed algorithm, $P_{j} \notin$ S_Discard ${ }^{c n}$. A contradiction.

Lemma 3. $N$ permanent checkpoints with the same checkpoint number form a globally consistent checkpoint.

Proof: We prove it by induction. In the beginning, the $N$ permanent ckpts with ckpt number 0 obviously form a globally consistent ckpt. Assume there are $N$ permanent ckpts with ckpt number $k$ and they form a globally consistent ckpt. In the proposed algorithm, if a process $P_{i}$ receives a message $m$ from another process $P_{j}$ during $I_{k}$, there are two possibilities:

Case 1: If $P_{j} \in S \_$Discard $^{k+1}$, there are two possibilities for $P_{j}$ :

1.1 $P_{j}$ does not receive DISCARD ${ }^{k+1}$ until $T_{\text {decide }}$ after entering $I_{k+1}$. From Section 4.3.2 we know $P_{j}$ 's local $M S S$ will set $\operatorname{RejectC} P_{j}$ to $k+1$, so that $P_{j}$ 's SoftCkpt ${ }^{k+1}$ will not be saved as PermCkpt $t^{k+1}$. It is $P_{j}^{\prime}$ 's Perm Ckpt be renumbered as PermCkpt $t^{k+1}$.

1.2 $P_{j}$ receives DISCARD ${ }^{k+1}$ before $T_{\text {decide }}$ after entering $I_{k+1}$. In this case, $P_{j}$ discards SoftCkpt ${ }^{k+1}$ and the preceding permanent ckpt PermCkpt ${ }^{k}$ of $P_{j}$ is renumbered as PermCkpt ${ }^{k+1}$.

From Lemma 2 we know $P_{i} \in S \_$Discard ${ }^{k+1}$. Through the above discussion, we know no matter if $P_{i}$ receives DISCARD ${ }^{k+1}$ or not, the preceding permanent ckpt PermCkpt $^{k}$ of $P_{i}$ is renumbered as PermCkpt ${ }^{k+1}$. Since the permanent ckpts with ckpt number $k$ form a globally consistent ckpt, there is no orphan message between the $k+1^{\text {th }}$ permanent ckpt of $P_{i}$ and the $k+1^{\text {th }}$ permanent ckpt of $P_{j}$.

Case 2: If $P_{j} \notin S \_$Discard ${ }^{k+1}, P_{j}$ does not receive DISCARD ${ }^{k+1}$ and its SoftCkpt $t^{k+1}$ is sent to HA/FA and saved as PermCkpt ${ }^{k+1}$. From the proposed algorithm, $P_{j}$ must send $m$ before it takes SoftCkpt ${ }^{k+1}$. Otherwise, $P_{i}$ will take Soft $\mathrm{Ckpt}^{k+1}$ before processing $m$, which makes $m$ been received within $P_{i}$ 's $I_{k+1}$. As a result, no matter $P_{i}$ 's PermCkpt $t^{k}$ is renumbered as PermCkpt ${ }^{k+1}$ or $P_{i}$ 's SoftCkpt ${ }^{k+1}$ is saved as PermCkpt $t^{k+1}$, there is no orphan message between $P_{i}$ 's PermCkpt ${ }^{k+1}$ and $P_{j}$ 's PermCkpt $t^{k+1}$.

Thus, if the $N$ permanent checkpoints with ckpt number $k$ form a globally consistent ckpt, there is no orphan message between the $k+1^{\text {th }}$ permanent ckpts of any two processes. That is, $N$ permanent ckpts with ckpt number $k+1$ form a globally consistent ckpt.
Theorem. The proposed algorithm always creates a consistent global checkpoint.

Proof: In the beginning there are $N$ permanent ckpts with ckpt number 0 , and they form the initial recovery line. Suppose there exists $N$ permanent ckpts with the same ckpt number $k$. In the proposed algorithm, we advance the recovery line to ckpt number $k+1$ only when all processes' permanent ckpts Perm Kpt $^{k+1}$ are collected. From Lemma 3, $N$ permanent ckpts with the same ckpt number form a globally consistent ckpt. Therefore, there always exists a consistent global ckpt.

\subsection{Performance Analysis}

In this section we discuss the performance of our checkpointing algorithm, including the blocking time, the number of permanent checkpoints, and the number of coordinating messages. Then we show the comparison with other protocols in a table. Here are the notations used in the following text:

- $N_{\min }$ : the number of processes that need to take checkpoints using the Koo-Toueg algorithm [1].

- $N_{\text {dep }}$ : the average number of processes on which a process depends. $\left(1 \leq N_{d e p} \leq N-1\right)$

- $C_{\text {wireless }}$ : cost of sending a message in the wireless link.

- $C_{\text {wired }}$ : cost of sending a message in the wired link.

- $C_{\text {broad }}$ : cost of broadcasting a message to all processes.

- $T_{c k p t}$ : the checkpointing time, including the delays incurred in transferring a checkpoint from a $M H$ to its $M S S$ and saving the checkpoint in the stable storage in the $M S S$ or a fixed host.

4.6.1. Blocking time. It is very clear that the blocking time of our protocol is 0 .

4.6.2. Number of new permanent checkpoints. In Section 4.3.3, we described that if there is an unacknowledged message like the scenario depicted in Figure 3, the MSS presumes the case in Figure 3(a) always occurs. That is, the receipt of message $m$ from $P_{j}$ is included in the Recv vector of $P_{i}$ 's $I_{c n-1}$. If it turns out later that Figure 3(b) is true instead, then there is a chance that $P_{j}$ and $P_{j}$-depended processes should not have been included in the dependency with the initiator. The consequence is that there may be additional soft checkpoints been made permanent, so as to increase the number of new permanent checkpoints. If we choose a proper $T_{\text {defer }}$ such that the untimely delayed $A C K \mathrm{~s}$ are very rare, the number of new permanent checkpoints is then close to minimum.

4.6.3. Number of coordinating messages. In the algorithm, the only coordinating message transmitted in the wireless link is the discard notification to a process in the set $S \_$Discard ${ }^{c n}$. The approximate number of discard 
notifications is $N-N_{\min }$. Messages sent in the wired link are $N$ Recv vectors from MSSs to $M S S_{\text {init }}$, and $N-N_{\text {min }}$ discard notifications from $M S S_{\text {init }}$ to $M S S$ s that serve the processes in $S \_$Discard $^{c n}$.

4.6.4. Comparison with other algorithms. Table 1 compares the performance of our algorithm with the algorithms in [1], [10], [12]. Compared to the Neves-Fuchs algorithm which is also time-based, our algorithm reduces the number of checkpoints to nearly minimum, so that the total number of checkpoints transmitted onto the fixed network is reduced. Fewer checkpoints transmitted also means less power consumption for mobile hosts. For a mobile computing system, it is also very critical to minimize the number and size of the messages transmitted in the wireless link. So, if we only consider the number of coordinating messages sent in the wireless link, our algorithm performs fairly well. For the size of the piggybacked information and the coordination message in the wireless link, our protocol outperforms Cao-Singhal algorithm with $O(1)$ to $O(N)$. On the other hand, the cost of transmitting a message in the wired link is far less than transmitting in the wireless link. So, although our protocol requires $O(N)$ coordinating messages in the wired network, the cost is affordable for wired networks with high bandwidth.

Table 1. Performance Comparison*

\begin{tabular}{|l|l|l|l|}
\hline Algorithm & $\begin{array}{l}\text { Blocking } \\
\text { time }\end{array}$ & $\begin{array}{l}\text { \# of } \\
\text { ckpts }\end{array}$ & \multicolumn{1}{|c|}{ \# of messages } \\
\hline $\begin{array}{l}\text { Koo-Toueg } \\
{[1]}\end{array}$ & $N_{\text {min }} \times T_{\text {ckpt }}$ & $N_{\text {min }}$ & $3 \times N_{\text {min }} \times N_{\text {dep }} \times\left(C_{\text {wired }}+C_{\text {wireless }}\right)$ \\
\hline $\begin{array}{l}\text { Neves-Fuchs } \\
{[12]}\end{array}$ & $\begin{array}{l}\sigma+2 \rho_{M H} T \\
-t d_{\text {min }}\end{array}$ & $N$ & $2 \times N \times C_{\text {wireless }}$ \\
\hline $\begin{array}{l}\text { Cao-Singhal } \\
{[10]}\end{array}$ & 0 & $N_{\text {min }}$ & $\begin{array}{l}\approx 2 \times N_{\min } \times\left(C_{\text {wired }}+C_{\text {wireless }}\right)+ \\
\min \left(N_{\text {min }} \times\left(C_{\text {wired }}+C_{\text {wireless }}\right), C_{\text {broad }}\right)\end{array}$ \\
\hline $\begin{array}{l}\text { Our } \\
\text { algorithm }\end{array}$ & 0 & $\begin{array}{l}\approx \\
N_{\text {min }}\end{array}$ & $\begin{array}{l}\approx\left(N-N_{\text {min }}\right) \times\left(C_{\text {wired }}+C_{\text {wireless }}\right)+ \\
N \times C_{\text {wired }}\end{array}$ \\
\hline
\end{tabular}

${ }^{*}$ The performance data of algorithms [1] and [10] are from [10].

\section{Conclusions}

In this paper we have proposed a time-based checkpointing protocol for mobile computing systems over Mobile IP. Our protocol reduces the number of checkpoints compared to the traditional time-based protocols. We also make use of the accurate timers in the $M S S$ s to adjust the timers in the $M H$ s, so that our protocol is well suited to mobile computing systems with $M H \mathrm{~s}$ spread across a wide area network. We also take advantage of the infrastructure provided by Mobile IP, so that the permanent checkpoints of the participating processes can be saved in the $H A$ or $F A$ depending on the process's current location. Compared to other protocols, our protocol performs very well in the aspects of minimizing the number and size of messages transmitted in the wireless media. Tracking and computing the dependency relationship between processes are performed in the MSSs, so that $M H$ s are free from additional tasks during checkpointing.

\section{Acknowledgement}

This research was supported in part by the Development of Communication Software Core Technology project of Institute for Information Industry and sponsored by MOEA, R.O.C.

\section{References}

[1] R. Koo and S. Toueg, "Checkpointing and RollbackRecovery for Distributed Systems," IEEE Trans. on Software Engineering, pp. 23-31, Jan. 1987.

[2] Z. Tong, R. Y. Kain, and W. T. Tsai, "A Low Overhead Checkpointing and Rollback Recovery Scheme for Distributed Systems," Proc. of the 8th Symp. on Reliable Distributed Systems, pp. 12-20, Oct. 1989.

[3] A. Acharya and B. R. Badrinath, "Checkpointing Distributed Applications on Mobile Computers," Proc. of Int'l Conf. on Parallel and Distributed Information Systems, pp. 73-80, Sep. 1994.

[4] R. Prakash and M. Singhal, "Low-Cost Checkpointing and Failure Recovery in Mobile Computing Systems," IEEE Trans. on Parallel and Distributed Systems, Vol. 7(10), pp. 1035-1048, Oct. 1996.

[5] N. Neves and W. K. Fuchs, "Adaptive Recovery for Mobile Environments," Comm. of the ACM, pp. 68-74, Jan. 1997.

[6] G. Cao and M. Singhal, "On the Impossibility of Min-Process Non-Blocking Checkpointing and An Efficient Checkpointing Algorithm for Mobile Computing Systems," Proc. of the $27^{\text {th }}$ Int'l Conf. on Parallel Processing, pp. 37-44, Aug. 1998.

[7] H. Higaki and M. Takizawa, "Checkpoint-Recovery Protocol for Reliable Mobile Systems," Proc. of the IEEE Symp. on Reliable Distributed Systems, pages 93-99, Oct. 1998.

[8] K. F. Ssu, B. Yao, W. K. Fuchs, N. Neves, "Adaptive Checkpointing with Storage Management for Mobile Environments,“ IEEE Trans. on Reliability, Vol. 48(4), pp. 315-324, Dec. 1999.

[9] T. Park and H. Y. Yeom, "An Asynchronous Recovery Scheme based on Optimistic Message Logging for Mobile Computing Systems," Proc. of the Int'l Conf. on Distributed Computing Systems, pp. 436-443, Apr. 2000.

[10] G. Cao and M. Singhal, "Mutable Checkpoints: A New Checkpointing Approach for Mobile Computing," IEEE Trans. on Parallel and Distributed Systems, Vol. 12(2), pp. 157-172, Feb. 2001.

[11] T. Park, N. Woo, and H. Y. Yeom, "An Efficient Recovery Scheme for Mobile Computing Environments," IEEE Int'l Conf. on Parallel and Distributed Systems, Jun. 2001.

[12] N. Neves and W. K. Fuchs, "Coordinated Checkpointing Without Direct Coordination," Proc. of the IEEE Int'l Computer Performance \& Dependability Symp., pp. 23-31, Sep. 1998. 